\title{
Effect of Mechanical Stimulation on Clinical and Radiographical Healing in a Post-Extraction Site
}

\author{
Barbato Luigi ${ }^{1}$, Gonnelli Alessio ${ }^{2 *}$, Baldi Niccolò ${ }^{3}$, Francioni Edoardo ${ }^{4}$ and Tonelli Paolo ${ }^{5}$ \\ 1 Unit of Oral Surgery, Department of Surgery and Tranlational Medicine, University of Florence Italy, \\ luigi.barbato1985@libero.it \\ 2 Unit of Oral Surgery, Department of Surgery and Tranlational Medicine, University of Florence Italy, \\ alessio.gonelli@me.com \\ 3 Unit of Oral Surgery, Department of Surgery and Tranlational Medicine, University of Florence Italy, \\ nicco.bald@gmail.com \\ 4 Unit of Oral Surgery, Department of Surgery and Tranlational Medicine, University of Florence Italy, \\ edoardo.francioni@alice.it \\ 5 Unit of Oral Surgery, Department of Surgery and Tranlational Medicine, University of Florence Italy, \\ paolo.tonelli@unifi.it \\ * Correspondence: alessio.gonelli@me.com; Tel.: +39 3396335656
}

\begin{abstract}
:
Aim: In a human pilot case, we used a root formed temporary prosthetical device (Intra-alveolar Device - IAD), to investigate the effect of mechanical stimulation on hard tissue clinically and radiographically. Background: The extraction of a tooth determines the resorption of the alveolar bone. This determines challenge to place an implant and to integrate the prosthesis in soft tissue. Mechanical stimulation is fundamental for bone tropism but is not understood the effect of mechanical stimulation on post-extraction site. Case presentation: A 24 years old woman was enrolled. The IAD's were used as expected by protocol. Clinical healing was uneventfully. Vertical radiographical bone resorption measured on $\mathrm{CBCT}$ was $-0,14 \mathrm{~mm}(5,6 \%)$ while horizontal bone resorption was $-0,21 \mathrm{~mm}(12,36 \%)$. Conclusion: This study demonstrated the feasibility of the proposed model. We need clinical prospectical studies enrolling more patients
\end{abstract}

Keywords: Bone resorption, Device, Tooth extraction

\section{Introduction}

The extraction of a tooth determines the resorption of the alveolar bone [1]. Different authors described this process during the last 50 years [2,3]. Mechanical stimulation is fundamental for bone tropism. ${ }^{4}$ The effect of mechanical stimulation on oral soft was investigated using a specific design of dental prosthesis intermediate element (ovate pontic) [5-6], however no author investigated the effect on bone post-extraction healing. In a human pilot case, we used a root formed temporary prosthetical device (Intra-Alveolar Device) (IAD) inserted in a post-extracion alveolous. Clinical and radiographical healing and the feasibility of the technique were evaluated.

\section{Case presentation}

A protocol was designed and approved by local ethical committee (Comitato Etico Area Vasta Centro - Rif. CEAVC 10206). The study was undertaken with the understanding and written consent of the participant and according to the principles of the Declaration of Helsinki. A woman 24 years old was enrolled. CBCT (Ortosophos XG, Sirona) was performed before the extraction (T0) and after 4 months (T1) using an individual stent. A field of view (FOV) of $8 \times 8 \mathrm{~cm}$, an exposure setting of $5.0 \mathrm{~mA} / 80 \mathrm{kV}$ and a scanning time of $17.5 \mathrm{sec}$ have been used. 


\section{Intra-Alveolar prosthetical device (IAD)}

DICOM data at T0 were segmented using the software Amira ${ }^{\circledR}$ to obtain the STL file of the tooth to be extracted. Using a 3D printer (Dimension Elite ${ }^{\circledast}$, Stratasys, resolution of $0,178 \mathrm{~mm}$ ) and the STL file, a replica of the tooth was printed in ABS resin. The replica was then transformed in acrylic resin due to problem of biocompatibility. Four copy of the IAD, with different design, were produced (Figure 1): IAD 1 was reduced circumferentially and vertically of the $25 \%$ of the root dimension, IAD 2 was reduced circumferentially and vertically of the $50 \%$ of the root dimension, IAD 3 was reduced circumferentially and vertically of the $70 \%$ of the root dimension, IAD 4 was reduce of the root part. The IADs were successively packaged and sterilized before the clinical use.

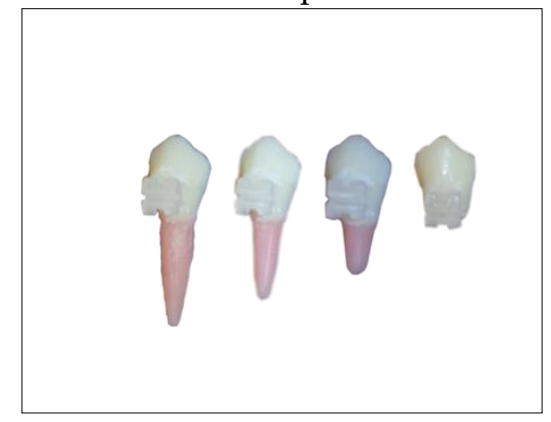

Figure 1: The figure shows 4 copies of IAD's after root part reduction

\section{Clinical procedure}

The right second mandibular premolar was extracted due to a diagnosis of vertical fracture. Post extraction socket was cleaned using a surgical spoon and irrigated with a physiological solution (Figure 2). The IAD 1 was inserted into the post-extractive socket. An altimeter was used to attach the brackets at the same height on the adjacent first premolar, first molar and on the IAD. The IAD 1 was splinted to the adjacent teeth using a rectangular steel arch. The prosthetical device was left in occlusion (Figure 2). Antibiotics (amoxicillin) and clorexidine mouth rinse were prescribed for a week. At 1 week, after a clinical exam, the IAD 1 was removed and the IAD 2 was splinted and inserted. At 2 weeks, after a clinical exam, the IAD 2 was removed and the IAD 3 was splinted and inserted. At 4 weeks, after a clinical exam, the IAD 3 was removed and the IAD 4 was splinted and inserted and left splinted for other 8 weeks. Clinical healing was uneventfully.

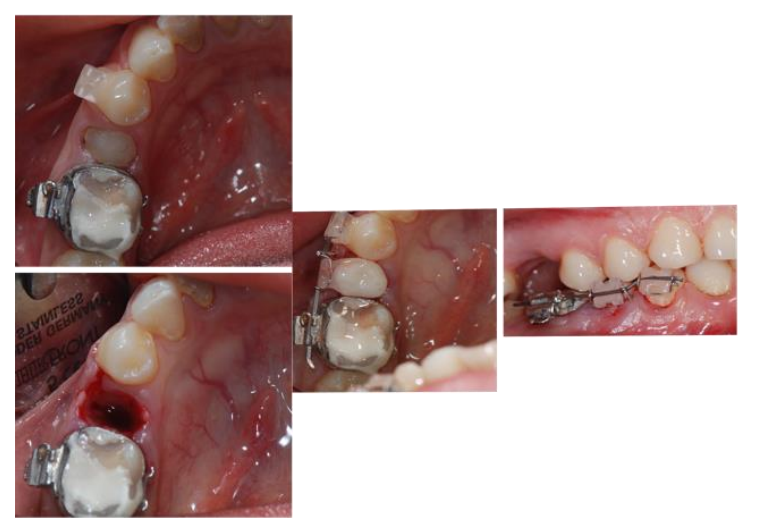

Figure 2: The figure shows the dental element before extraction, the alveolus after extraction and IAD in postextractive alveolus 


\section{Radiographical healing}

DICOM data (Digital Imaging and Communications in Medicine), retrieved from CBCT at T0 and T1, were segmented by a software (Amira ${ }^{\circledR}$ ) to obtain two STL (Standard Triangulation Language) files. The STL files were matched using Di2Mesh ${ }^{\circledR}$ software. The superimposed color-coded figure was obtained by matching the STL file at $\mathrm{T} 0$ and at $\mathrm{T} 1$ and showed green area in post extractive site corresponding to bone resorption comprised between 0 and $1 \mathrm{~mm}$ (Figure 3).

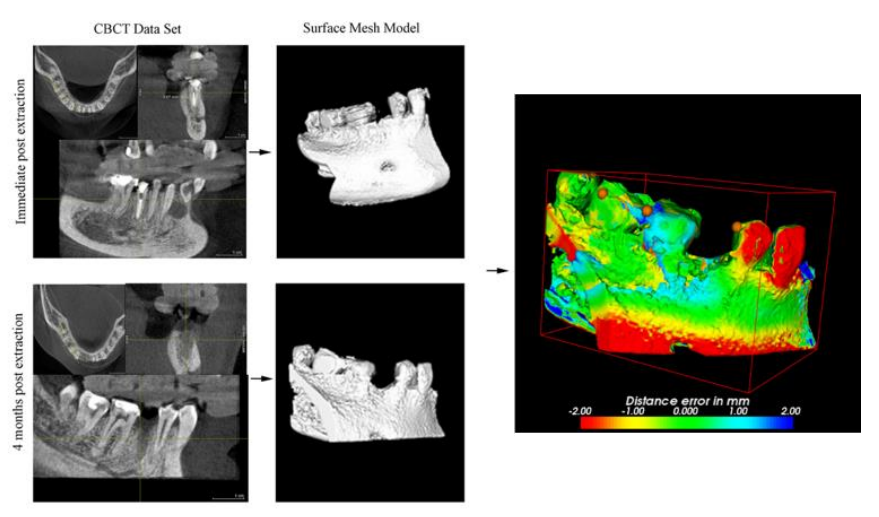

Figure 3: Superimposed color-coded figure was obtained by matching the STL file at T0 and at T1.

The alveolar bone resorption was also measured on CBCT using a technique already described in literature [7]. Data on quantitative resorption are reported in table 1.

\begin{tabular}{|l|l|l|l|l|}
\hline \multicolumn{5}{|c|}{ Table 1 } \\
\hline & T0 & T1 & Difference & Difference \% \\
\hline L1-V1 & $2,49 \mathrm{~mm}$ & $2,35 \mathrm{~mm}$ & 0,14 & $5,6 \%$ \\
A-A1 & $0,6 \mathrm{~mm}$ & $0,53 \mathrm{~mm}$ & 0,07 & $11,6 \%$ \\
\hline B-B1 & $1,49 \mathrm{~mm}$ & $1,3 \mathrm{~mm}$ & 0,19 & $12,7 \%$ \\
\hline C-C1 & $2,73 \mathrm{~mm}$ & $2,38 \mathrm{~mm}$ & 0,35 & $12,8 \%$ \\
\hline Horizontal average & & & 0,21 & $12,36 \%$ \\
\hline
\end{tabular}

Tab.1. Linear measurement on CBCT

\section{Discussion}

Tissue differentiation is mechanobiologically regulated. Using as model the mechanical stimulation of the fracture healing process, some authors reported positive effects on the bone regeneration process while other authors reported negative ones [8]. For the post-extraction alveolus no author has investigated in vivo the effect of the mechanical stimulation on the bone healing process. Only few authors analysed its effect on oral soft tissue [6]. In this human pilot study the possibility to use an IAD following the extraction of a tooth was investigated. The IAD was realized in acrylic resin that has not osteogenetic, osteoinductive and osteconductive properties and was used to avoid interferences on bone healing. We found horizontal bone loss $(0,21 \mathrm{~mm}$ corresponding to the 
$12,36 \%)$ higher than vertical bone loss $(0,14 \mathrm{~mm}$ corresponding to the $5,6 \%)$ four months after extraction. Bone resorption at 4 months using the IAD is lower than bone resorption obtained by spontaneous healing of post extraction socket described by Araujo et coll. (50\% of original dimension) [1]. We treat only a patient so data are not significative, however the IAD has been clinical safe and the bone healing after 12 weeks was uneventfully. Principal limit was represented by IAD's substitution, which increased chair time. The IAD's can't be printed directly in acrylic resin, furthermore this protocol cannot be used in case of adjacent multiple edentulous site. In order to evaluate the feasibility of this technique, we need clinical prospectical studies enrolling more patients. Moreover the healing of the post extraction alveolus stimulated by the IAD should be analysed also from histological, histomorphometrical and immunohistochemical ways.

\section{Conclusions}

The IAD can be used to reduce bone resorption after extraction of a tooth and at the same time can be used to reduce the funzional deficits following tooth extraction. The IAD can prove the importance of the mechanical stimulation on the bone healing process and in the ridge preservation following tooth extraction. This study demonstrated the feasibility of the proposed model. We need clinical prospectical studies enrolling more patients

Funding: This research received no external funding

Conflicts of Interest: The authors declare no conflict of interest

Acknowledgments: We thank Dr. Silvia Pancani for kind support.

\section{References}

1. Araùjo, M.G.; Silva, O.C.; Misawa, M.; Sukekava, F. Alveolar socket healing: what can we learn. J Clin Periodontol. 2016; 68,122-134.

2. Schropp, L.; Wenzel, A.; Kostopoulos, L.; Karring, T. Bone healing and soft tissue contour changes following single-tooth extraction: a clinical and radiographic 12-month prospective study. Int J Periodontics Restorative Dent. 2003; 23(4),313-323.

3. Trombelli, L.; Farina, R.; Marzola, A.; Bozzi, L.; Liljenberg, B.; Lindhe, J. Modeling and remodeling of human extraction sockets. J Clin Periodontol. 2003; 35(7),630-639.

4. Tyrovola, J.B. The "Mechanostat Theory" of Frost and the OPG/RANKL/RANK System. J Cell Biochem. 2015; 116(12),2724-2729.

5. Orsini, G.; Murmura, G.; Artese, L.; Piattelli, A.; Piccirilli, M.; Caputi, S. Tissue healing under provisional restorations with ovate pontics : A pilot human histological study. J Prosthet Dent. 2015; 96(4),252- 257

6. Zitzmann, N.U.; Marinello, C.P.; Berglundh T. The ovate pontic design: a histologic observation in humans. J Prosthet Dent. 2015; 88(4),375-380.

7. Chappuis, V.; Engel, O.; Reyes, M., Shahim, K.; Nolte, L.P.; Buser, D. Ridge alterations postextraction in the esthetic zone: a 3D analysis with CBCT. J Dent Res. 2013; 92(12 Suppl),195-201.

8. Betts, D.C.; Müller, R. Mechanical regulation of bone regeneration: theories, models, and experiments. Front Endocrinol (Lausanne). 2014; 5,211. 